\title{
Ontological issues in distance perception: Cue use under full cue conditions cannot be inferred from use under controlled conditions
}

\author{
Mark Mon-WiLliams \\ University of Aberdeen, Aberdeen, Scotland \\ AND \\ GeOFFREY P. BinghaM \\ Indiana University, Bloomington, Indiana
}

\begin{abstract}
Phosphorescent square tiles (arranged to yield a single image size) were viewed in the dark by 56 monocular observers who utilized a chinrest. The targets were placed at one of three horizontal distances and at one of three eye heights, allowing us to study the relative effect of height in the visual field (HVF) and sagittal distance on observers' verbal reports of the horizontal distance at which the object lay (near, middle, or far). In Experiment 1, we found that reports covaried primarily with HVF and, as predicted, they exhibited a weak paradoxical inverse relation with horizontal distance. In a second and third experiment, a visible surface was placed under the targets at the three eye heights in both dark and fully lighted conditions. In this situation, the inverse distance relation disappeared, and HVF no longer influenced the judgments of most observers. The results show that information projected from relevant support surfaces is essential for veridical information about object distance. These results raise fundamental issues for perceptual researchers regarding how to decide when a cue has been properly delineated, given the assumption that the relation between a cue and what it specifies is probabilistic.
\end{abstract}

The study of visual perception requires the investigator to make a hypothesis about the optical information or cue that is detected by the visual system when perceiving a given property of the world. Part of the challenge is that there may be a number of different potential cues or sources of information about a given property. A classic example is distance perception, for which a rather extensive list of cues has been developed, including motion parallax, binocular vergence, image size of familiar objects, and height in the visual field, among many others. Once an investigator has formulated a potential cue, the problem is to determine whether in fact that cue is detected and used by the visual system. Especially in the case of distance perception, it is generally accepted that multiple cues are detected and used simultaneously to perceive distance. Nonetheless, the different cues vary in terms of the precision of the information they provide; thus, it is expected that the cues will be differentially weighted when combined to determine a perceived distance (see, e.g., Bruno \& Cutting, 1988; Cutting \& Vishton, 1995; Landy, Maloney, Johnston, \& Young, 1995). In other words, the relative contribution of a cue is expected to vary. Thus, if the information provided by a cue diverges somewhat from the information provided by other cues, then it is not surprising when the perceived distance is not exactly the same as that specified by the given cue.

There is a major problem posed by this circumstance for perceptual theory and methods of investigation. The problem lies in the formulation of a given cue. How does the investigator know when he or she has it right? The effort in describing cues is to differentiate aspects of optical pattern and/or eye postures and movements that reliably covary with the property of the world to be perceived. The question is, when does an aspect of optical pattern constitute a differentiated information variable for the visual system? A potential problem is that a pattern described as a cue might be incompletely specified. That is, the cue is not really a cue at all but is an aspect of a larger pattern to which the visual system normally responds. Because the differentiated aspect is, in fact, a part of the relevant information variable, it may successfully stimulate the visual system, and the investigator who expects only a partial response interprets the results as confirming his or her hypothesis that the incomplete pattern is a cue normally detected and used by the visual system.

The standard methods of investigation are to first test a hypothesized cue in isolation in order to see whether observers can successfully detect the cue to perceive the 
given property. When a partial pattern is isolated, the visual system may respond to it, although the response might be weak or inaccurate. Once sensitivity to a variable has been shown, then the cue can be tested in combination with others in circumstances that are more representative of normal performance conditions (see, e.g., Epstein, 1966; Landy et al., 1995; Philbeck \& Loomis, 1997; Wallach \& O'Leary, 1982). When a partial pattern is tested in the context of a larger pattern (i.e., more information), then an incomplete variable covaries with the rest and is thus interpreted as partially contributing to the response. Using perturbation methods, the cue can be manipulated to determine the extent to which the resulting perceptually guided performance (judgments or actions) varies with the information variable of interest (e.g., Landy et al., 1995). To reiterate, because a partially specified pattern would covary with a cue that the visual system normally uses, the visual system might respond to the manipulation of that pattern, although weakly so. The variable is then interpreted as having a low weighting - that is, normally having a small effect. The question is, what can be safely concluded from the results when we test the visual system under conditions in which we isolate a hypothetical information variable (whether it is absolutely isolated or isolated only in the sense that it alone is being manipulated)? The problem is that the visual system is highly adaptive; thus, it can respond in perturbed conditions in ways that do not reflect its normal behavior.

In the present article, we set out to illustrate this theoretical and methodological problem by investigating potential incomplete information variables, or cues, to discover whether observers can be found to use a variable reliably in conditions that isolate it and then fail to use the variable at all under more representative conditions. We selected a classic cue - height in the visual field (HVF) - to investigate this issue (see, e.g., Cutting, 2003; Cutting \& Vishton, 1995; Gillam, 1995). Evidence in the literature indicates that HVF is salient and effective information about distance (Cutting \& Vishton, 1995). In the context of more than one kind of task, researchers have suggested that it provides useful information to the observer (Gardner \& Mon-Williams, 2001; Mon-Williams, McIntosh, \& Milner, 2001; Ooi, Wu, \& He, 2001; Philbeck \& Loomis, 1997).

HVF has also been called elevation (Collett \& Udin, 1988; Philbeck \& Loomis, 1997), vertical gaze angle (Gardner \& Mon-Williams, 2001; Mon-Williams et al., 2001), or slope of regard (see, e.g., Wallach \& O'Leary, 1982). With these variations in the label, there have also been variations in the description of the information. HVF is sometimes described explicitly with reference to a support or ground surface (e.g., Sedgwick, 1986). Given the geometry of viewing objects distributed at different distances along a level ground surface, the images of those objects will appear at increasing height in the visual field with increasing distance. The limit of the ground surface is the horizon. HVF is sometimes described in comparison with the image of the horizon (Gillam, 1995; Sedgwick, 1986). Vertical gaze angle and slope of regard (and sometimes also elevation) are described in terms of the angle between a horizontal and a line of gaze or sight (Gardner \& Mon-Williams, 2001; Mon-Williams et al., 2001; Wallach \& O'Leary, 1982). Reference to a support surface is typically implicit or absent in such accounts. Often, these different labels are used interchangeably (e.g., Gardner \& Mon-Williams, 2001; Philbeck \& Loomis, 1997; Schiff, 1980).

In the present experiments, we test HVF in its vertical gaze angle (or slope of regard or elevation) formulation - that is, as the vertical angle between a horizontal and the line of sight from the eye to a target object. A large angle specifies a nearby object, and a small angle specifies a more distant object. As shown in Figure 1, we set up a configuration of seven target objects that were at one of three different horizontal distances from the observer and at one of three different heights in the visual field. By varying the eye height of the targets using three different eye heights, we were able to decouple variations in horizontal distance and HVF. Accordingly, among the seven target locations, there were three that varied HVF at constant distance and three that varied distance at constant HVF. These configurations shared a common target location; thus, together these composed five target locations. We used these five targets to test the extent to which judgments of distance covaried with HVF or with distance, because this particular configuration tested each of the variables independently of the other. We had observers gauge all seven locations so that there would be multiple targets at each horizontal distance. We did not test all three eye heights at each distance (i.e., nine target locations), because this would have added additional HVF (more than three). However, in Experiment 3, we did test the nine-target configuration as a control experiment.

In successive experiments, we tested distance judgments in isolated (or reduced) cue conditions in the dark, then in conditions that included visible support surfaces

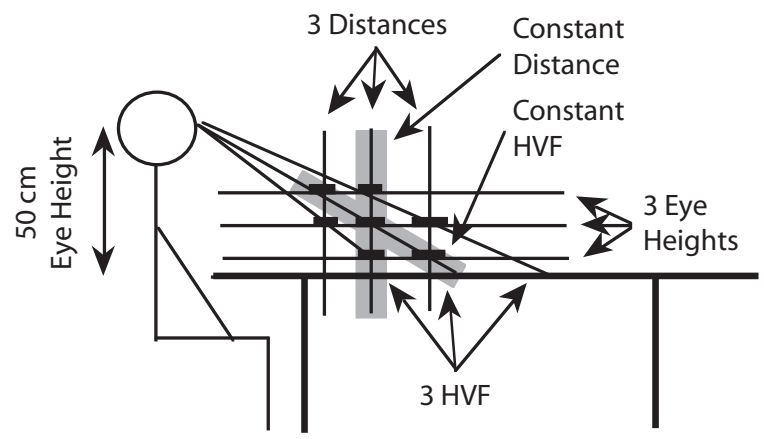

Figure 1. Arrangement of the targets in Experiments 1 and 2 showing how covariation of horizontal distance from the observer and eye height yielded variations in HVF that were uncorrelated with target distance. The seven target locations shown were judged by observers. Analyses were performed on judgments of five target locations shown in gray bands. One band contains targets at three distances but one $\mathrm{HVF}$, and the other band contains targets at three HVF and one distance. 
in the dark and, finally, in conditions that included visible support surfaces in normal room illumination. The expectation was that observers would use HVF to gauge distance in the isolated cue condition; thus, they would be entirely incorrect in their reports of distance. Furthermore, as information about the support surfaces was introduced, observers would use different surface-relative information to gauge distances correctly; thus, their reports would cease to covary with HVF. That is, observers would use HVF in the first case because that was (nearly) all that they had available, then they would not use it once additional information was introduced. These different conditions were tested between subjects, and no feedback was provided in any condition. If the expectations were met, then the isolated cue performance could not be used to predict the subsequent performance under more representative conditions. The full theoretical and methodological implications of these expected results are addressed in the General Discussion.

\section{EXPERIMENT 1}

We tested the use of HVF isolated in reduced viewing conditions that eliminated other potential sources of information about target distances. Cutting and Vishton (1995) highlighted four assumptions that must be met in order for HVF to provide metric information regard- ing an object's distance. These assumptions are (1) that the ground plane is opaque, (2) that gravity constrains each object to have its base on the surface of support, (3) that the observer's eye is at a known distance above the surface of support, and (4) that the surface of support is generally planar and orthogonal to gravity. Cutting and Vishton (1995, p. 86) suggested that only the first two assumptions are reasonable; thus, the role of HVF is restricted to providing "good ordinal information about distance from the point of observation." In Experiment 1, we were interested in the issue of how participants would respond when there was no information that could allow them to verify the validity of any "assumptions" regarding the information conveyed by HVF. It was possible that the participants might ignore the changes in HVF that occurred within our design. Nevertheless, we hypothesized that participants would latch onto this signal - in part, because it would be the only salient visual variable in the display. The displays were also designed to violate all of the assumptions listed by Cutting and Vishton, with the result that there was no correlation between HVF and distance. If participants used HVF to gauge distance, then they would report distance incorrectly. In fact, their reports would fail to correlate with distance at all.

Finally, there was a potential source of information that remained in addition to HVF because it could not be
A
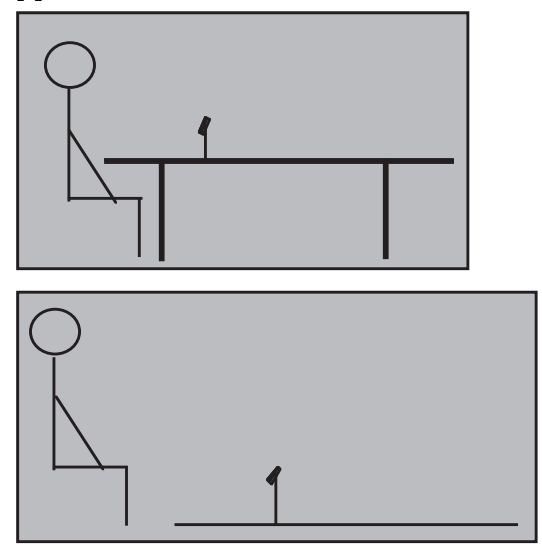

C

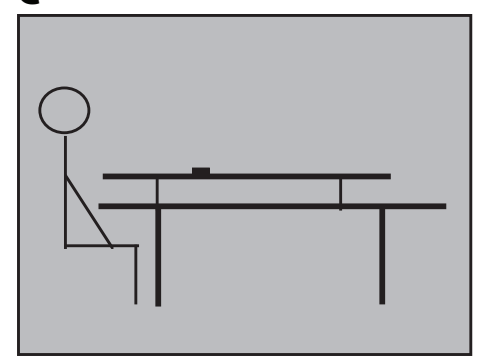

B
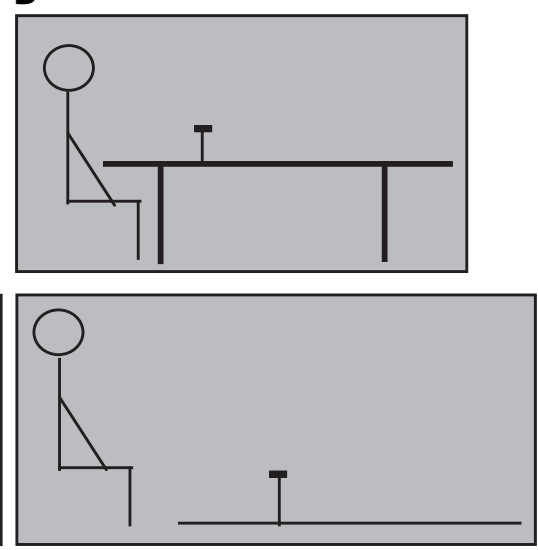

D

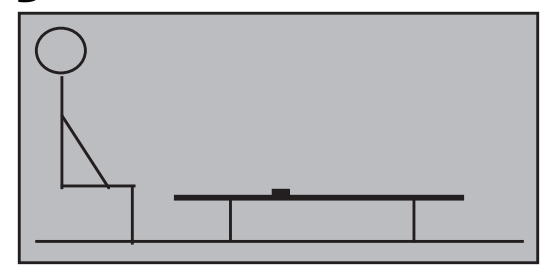

Figure 2. Illustration of the various conditions tested in the present experiments. (A) Frontoparallel targets supported at $50-\mathrm{cm}$ and then at $100-\mathrm{cm}$ eye heights. (B) The same two eye heights for horizontal targets. (C) A target on a support surface itself supported at a 50-cm eye height. (D) The same as that in (C) at 100-cm eye height. 
removed from the displays. This other cue was accommodation. It is known that changes in accommodation can alter participants' pointing responses in reduced cue environments so that altering focus from a far object to a near object causes participants to point closer (Fisher \& Ciuffreda, 1988; Mon-Williams \& Tresilian, 2000). Analysis of the alterations in pointing response suggests that accommodation can provide ordinal distance information to the human observer, but it is too coarse a cue to provide useful metric information (Mon-Williams \& Tresilian, 2000). The influence of accommodation in verbal reports of distance is not predicted from its influence on pointing responses. Mon-Williams and Tresilian (1999) showed that participants provide verbal reports in the opposite direction of their pointing response in sparse viewing environments in which the image size is kept constant. The key to understanding this phenomenon lies in both the presence of size constancy mechanisms and the fact that distance perception for the purpose of motor control is not cognitively penetrable. It has been known since the time of Emmert (1881) that perceived size is a function of perceived distance. In the experiment of Mon-Williams and Tresilian (1999), a luminous object was viewed in darkness while a prism was placed in front of one eye to either increase or decrease vergence angle. Increased vergence angle signaled that the object was closer, and this caused participants to underestimate distance in an openloop pointing task (the opposite effect was found with decreased vergence angle). It follows from Emmert's law, however, that increasing vergence angle causes the object to be perceived as smaller (because objects normally increase their retinal extent as they approach an observer). In line with this, participants reported that the object was smaller in the study of Mon-Williams and Tresilian (1999). The decrease in perceived size had the additional effect of causing participants to report that the object was farther away. This effect arises because the only cognitively penetrable distance information available to the participants is image size. Smaller objects are normally farther away, and this influenced the participants' verbal reports. Thus, the participants showed dissociation between their verbal reports and their motor responses. This effect is known as the size-distance paradox effect. These findings led us to predict that our participants would report closer targets as being farther away and vice versa.

\section{Method}

Participants. Sixteen adults - aged 18-27 years - participated as observers, 8 in each of two conditions. All had normal or correctedto-normal vision. All were reimbursed at $\$ 10$ per hour.

Apparatus and Procedure. Targets were square tiles that were painted black and covered on the top with green triangular phosphorescent texture elements. As shown in Figures 2A and 2B, tiles were attached with Velcro to a little, black wooden platform that rested at the appropriate orientation on black rods of one of three lengths that were each inserted into a black wooden base of support. Only the phosphorescent surface texture on the tile could be seen in the dark. Three different tiles - each with different texture elements - were used for each size and orientation, and each time they were attached to the platform, they were rotated so that a different edge was up, thus changing the orientation of the texture pattern. Therefore, the same particular tile and texture pattern was not seen more than once at a given location. The supports were placed either on a table (as shown in the top panels of Figures $2 \mathrm{~A}$ and $2 \mathrm{~B}$ ) or on the floor (as shown in the bottom panels of Figures $2 \mathrm{~A}$ and $2 \mathrm{~B}$ ). The table or floor surfaces were covered with black felt.

As shown in Figure 1, seven target locations included three distances from the observer's chest or eye plane (horizontal distance), three eye heights, and three HVF. Eye-height variations yielded three target locations that varied in horizontal distance but not in HVF, and three target locations that varied in HVF but not in horizontal distance. The two sets shared a single target location so that the two sets comprised five target locations. The two sets were uncorrelated with respect to HVF and horizontal distance. The average target size was $7 \mathrm{~cm}$, but target size (and triangular texture element size and density) covaried with viewing distance in order to hold image size constant. There were three differently textured targets of each size that were randomly selected and rotated at each presentation so that no target could be recognized from the texture pattern over repeated trials at a given target location. Two conditions were tested within subjects. In one, tiles were oriented to lie perpendicular

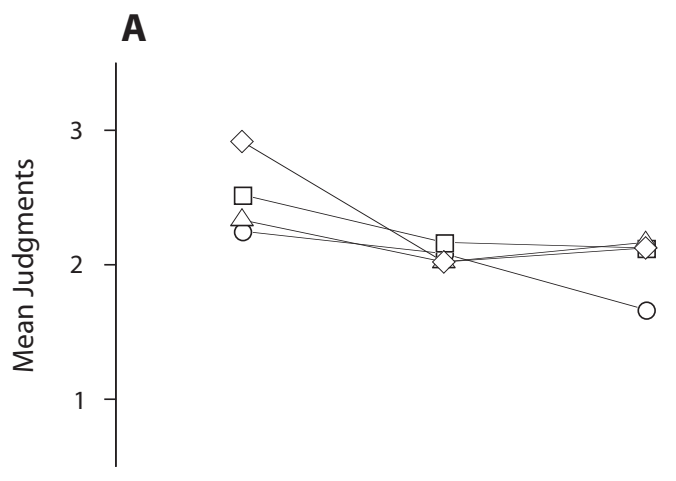

1

2

3

B

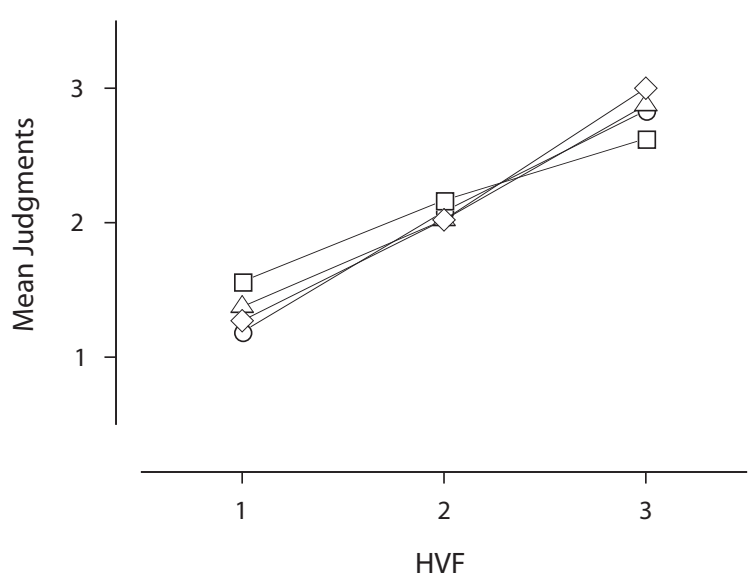

Figure 3. Results of Experiment 1 with isolated targets in the dark. (A) Judgment means for three targets at a single HVF as a function of horizontal distance. (B) Judgment means for three targets at a single horizontal distance as a function of HVF. Frontoparallel targets at $50 \mathrm{~cm}$ (open circles) and $100 \mathrm{~cm}$ (open triangles), and horizontal targets at $50 \mathrm{~cm}$ (open squares) and $100 \mathrm{~cm}$ (open diamonds). 
to sight lines - that is, in frontoparallel planes. In the other, targets were oriented horizontally - that is, as if lying on a support surface. In this latter condition, tile surface slant was constant for a given HVF, but it varied with horizontal distance. Subsequently, the horizontal orientation of tiles would be required to place them on visible surfaces. Thus, we tested whether observers would detect local slant variations and use them to begin to discriminate actual horizontal distances.

Targets were viewed in the dark by monocular observers (wearing an eye patch) who rested their head on a chinrest. In order to control potential effects of accommodation, two eye-height ranges were tested. Target supports were placed on a table at a $50-\mathrm{cm}$ eye height or on the floor at a $100-\mathrm{cm}$ eye height. For the $50-\mathrm{cm}$ range, target eye heights were $22.5,30$, and $40 \mathrm{~cm}$, and horizontal target distances were $20,26.7$, and $35.5 \mathrm{~cm}$. For the $100-\mathrm{cm}$ range, target eye heights were 50,67 , and $90 \mathrm{~cm}$, and horizontal target distances were 45 , 60 , and $80 \mathrm{~cm}$. In both cases, HVF were $33.7^{\circ}, 41.6^{\circ}$, and $49.9^{\circ}$. (Note: We label the two eye-height ranges as 50 and $100 \mathrm{~cm}$, but there were three different eye heights tested at each of these ranges.) Small pieces of cardboard were placed under the felt surfaces so the experimenter could place the targets accurately by feeling the raised loci through the felt.

Observers reported horizontal distances as being near, medium, or far from their chest. The experimenter illustrated the three distances to the observers by holding his hand out in front of the observer's chest successively at each of the three distances. This was done under normal room lighting. Participants did not see the presentation apparatus (targets, table, etc.) in normal room lighting before the experiment. Participants were told nothing about the number of targets or their locations. They were only told to report at which of the three distances each target lay. The seven target locations were tested in a random order within each of five repeated blocks of trials in each condition. One group of observers was tested at the $50-\mathrm{cm}$ range, and the other was tested at the $100-\mathrm{cm}$ range. For each group, all the frontoparallel targets were tested first, followed by all the horizontal targets. There were no practice trials.

The participants lowered occluding goggles between trials while the experimenter was positioning targets. The participants then raised the goggles in order to view the target and make a report.

The full design included one between-subjects factor-eyeheight range $(50 \mathrm{~cm}$ and $100 \mathrm{~cm})$ - and four within-subjects fac-
Table 1

Results of Multiple Regressions Performed on Group Data in Four Conditions of Experiment 1

\begin{tabular}{ccccccr}
\hline \multirow{2}{*}{$\begin{array}{c}\text { Eye } \\
\text { Height }\end{array}$} & \multirow{2}{*}{$R^{2}$} & \multicolumn{2}{c}{ HVF } & & \multicolumn{2}{c}{ Distance } \\
\cline { 7 - 7 } \cline { 5 - 6 } & & Coefficient & Partial $F$ & & Coefficient & Partial $F$ \\
\hline $50 \mathrm{~cm}$ & & & & & & \\
FPT & $.54^{* * *}$ & $.83^{* * *}$ & 166.2 & & $-.30^{* * *}$ & 21.4 \\
HT & $.25^{* * *}$ & $.53^{* * *}$ & 46.7 & & $-.20^{*}$ & 6.5 \\
$100 \mathrm{~cm}$ & & & & & \\
FPT & $.41^{* * *}$ & $.75^{* * *}$ & 107.6 & & -.09 & 1.7 \\
HT & $.68^{* * *}$ & $.86^{* * *}$ & 280.9 & & $-.39^{* * *}$ & 58.0 \\
\hline
\end{tabular}

Note-FPT, frontoparallel tile orientation; HT, horizontal tile orientation; HVF, height in the visual field. ${ }^{*} p<.05 .{ }^{* * *} p<.001$.

tors: tile orientation (frontoparallel and horizontal), horizontal distance (near, medium, and far), HVF (low, medium, high), and repetition (1-5).

\section{Results}

Although seven target locations were judged, we analyzed and reported the data only from the five target locations that yielded constant horizontal distance and three HVF, and constant HVF and three horizontal distances. First, we assigned near reports as 1, medium as 2, and far as 3 ; then, we computed means for each location. The means for frontoparallel-oriented and horizontal-oriented targets at each of two eye-height ranges are shown in Figure 3. Panel A shows means for three targets at constant HVF but different horizontal distances. Panel B shows means for three targets at constant horizontal distance but different HVF. In line with the predictions, the mean reports varied with HVF, as was expected in all four cases. Reports also varied inversely with distance-as was expectedalthough weakly so. The results confirmed our expectation that reports would covary with HVF; thus, judgments of distance would be incorrect. Target orientation (frontopar-

Table 2

Results of Multiple Regressions Performed on Individual Observer Data in Four Conditions of Experiment 1

\begin{tabular}{|c|c|c|c|c|c|c|c|c|c|c|c|}
\hline \multicolumn{6}{|c|}{ 50-cm Eye Height } & \multicolumn{6}{|c|}{ 100-cm Eye Height } \\
\hline \multirow{2}{*}{$\begin{array}{l}\text { NS } \\
\text { Factor }\end{array}$} & \multirow[b]{2}{*}{$R^{2}$} & \multicolumn{2}{|c|}{ HVF } & \multicolumn{2}{|c|}{ Distance } & \multirow{2}{*}{$\begin{array}{c}\text { NS } \\
\text { Factor }\end{array}$} & \multirow[b]{2}{*}{$R^{2}$} & \multicolumn{2}{|c|}{ HVF } & \multicolumn{2}{|c|}{ Distance } \\
\hline & & Coefficient & Partial $F$ & Coefficient & Partial $F$ & & & Coefficient & Partial $F$ & Coefficient & Partial $F$ \\
\hline \multicolumn{6}{|c|}{ Frontoparallel Targets } & \multicolumn{6}{|c|}{ Frontoparallel Targets } \\
\hline $\mathrm{X}$ & $.91^{* * *}$ & $1.0^{* * *}$ & 164.8 & -.12 & 2.5 & & .19 & .50 & 3.8 & -.12 & 0.2 \\
\hline & $.63^{* * *}$ & $.88^{* * *}$ & 21.6 & $-.50^{*}$ & 7.0 & $X$ & $.59^{* * *}$ & $.88^{* * *}$ & 24.2 & -.12 & 0.5 \\
\hline $\mathrm{X}$ & $.77^{* * * *}$ & $1.0^{* * *}$ & 56.1 & -.12 & 0.8 & $\mathrm{X}$ & $.40^{*}$ & $.75^{* *}$ & 10.1 & .25 & 1.1 \\
\hline $\mathrm{X}$ & $.69^{* * *}$ & $1.0^{* * *}$ & 37.5 & -.12 & 0.5 & $X$ & $.35^{*}$ & $.75^{* *}$ & 8.9 & .12 & 0.3 \\
\hline \multirow[t]{4}{*}{$\mathrm{X}$} & $.72^{* * *}$ & $1.0^{* * *}$ & 41.2 & -.25 & 2.5 & $\mathrm{X}$ & $.70^{* * *}$ & $.88^{* * *}$ & 38.5 & -.12 & 0.8 \\
\hline & .18 & -.12 & 0.4 & -.38 & 3.3 & $\mathrm{X}$ & $.36^{*}$ & $.62^{* *}$ & 9.3 & -.12 & 0.4 \\
\hline & $.77^{* * *}$ & $.88^{* * *}$ & 42.9 & $-.50^{* *}$ & 14.0 & $X$ & $.58^{* * *}$ & $.88^{* * *}$ & 22.9 & -.12 & 0.5 \\
\hline & $.86^{* * *}$ & $1.0^{* * *}$ & 95.4 & $-.38^{* *}$ & 13.4 & & $.79^{* * *}$ & $.75^{* * *}$ & 45.0 & $-.50^{* * *}$ & 20.0 \\
\hline \multicolumn{6}{|c|}{ Horizontal Targets } & \multicolumn{6}{|c|}{ Horizontal Targets } \\
\hline & $.59^{* * *}$ & $.62^{* * *}$ & 18.0 & $-.38^{*}$ & 6.4 & & $.72^{* * * *}$ & $.88^{* * *}$ & 33.8 & $-.50^{* *}$ & 11.0 \\
\hline $\mathrm{X}$ & $.57^{* * *}$ & $1.0^{* * *}$ & 23.6 & .14 & 0.5 & & $.66^{* * *}$ & $.75^{* * *}$ & 26.1 & $-.38^{*}$ & 6.5 \\
\hline \multirow[t]{2}{*}{$\mathrm{X}$} & $.50^{* *}$ & $.50^{* *}$ & 13.9 & -.25 & 3.5 & $X$ & $.77^{* * *}$ & $1.0^{* * *}$ & 56.0 & -.12 & 0.9 \\
\hline & .04 & .12 & 0.3 & -.12 & 0.3 & & $.89^{* * *}$ & $1.0^{* * *}$ & 113.3 & $-.50^{* * *}$ & 28.3 \\
\hline \multirow[t]{2}{*}{$X$} & $40^{*}$ & $.62^{* *}$ & 8.4 & -.38 & 3.0 & $\mathrm{X}$ & $.53^{* * *}$ & $.88^{* * *}$ & 17.6 & -.25 & 1.4 \\
\hline & .17 & .12 & 0.6 & .25 & 2.7 & & $.71^{* *}$ & $.75^{* * *}$ & 28.3 & $-.50^{* *}$ & 12.6 \\
\hline \multirow[t]{2}{*}{$X$} & $.56^{* * *}$ & $.75^{* * *}$ & 19.4 & -.25 & 2.1 & & $.80^{* * * *}$ & $.88^{* * *}$ & 50.2 & $-.50^{* * *}$ & 16.4 \\
\hline & $.56^{* * *}$ & $.50^{* *}$ & 8.3 & $-.62^{* *}$ & 13.0 & & $.64^{* * * *}$ & $.75^{* * *}$ & 24.1 & $-.38^{*}$ & 6.0 \\
\hline
\end{tabular}

Note-HVF, height in the visual field. ${ }^{*} p<.05 .{ }^{* *} p<.01 .{ }^{* * *} p<.001$. 
allel or horizontal) had no effect, so observers did not use local slant variations to detect horizontal distances.

We performed a multiple regression on the combined observer data for each condition regressing HVF and frontoparallel distance on verbally reported distance. The results are shown in Table 1, in which it can be seen that the inverse relation of judgments to horizontal distance was significant in three of the four conditions. We performed this analysis separately on the data for each participant. As shown in Table 2, the inverse distance judgments were significant for less than half of the observers only. Judgments varied strictly with HVF and not with actual horizontal distance for at least half of the observers in all conditions, except 100 -cm eye height with horizontal targets, in which case, the inverse distance judgments were prevalent. Nevertheless, judgment varied strongly with HVF. The orientation of the targets did not appear to be a significant manipulation. In principle, the horizontal orientation of the square tiles might have begun to provide information about horizontal distance as would a visible support surface, whereas frontoparallel targets would have strictly isolated HVF. In practice, the orientation of the targets did not appear to be a significant manipulation.

\section{EXPERIMENT 2}

In Experiment 2, we tested the influence of HVF when horizontal targets rested on a visible support surface at each of three eye heights. The surface was $30 \mathrm{~cm}$ wide and $1 \mathrm{~m}$ long and was covered with a phosphorescent checkerboard pattern (2-cm squares). We tested both the $50-\mathrm{cm}$ and $100-\mathrm{cm}$ eye-height ranges. At the $50-\mathrm{cm}$ eyeheight range, we also tested performance under conditions of normal room illumination. Finally, at the $100-\mathrm{cm}$ eye-height range, we also tested a constant image size checkerboard surface viewed in the dark (i.e., three surfaces, one for each eye height). The highest and lowest surfaces and the texture thereon varied in size as compared with the $30-\mathrm{cm} \times 100$ - $\mathrm{cm}$ size of the middle surface in order to preserve constant image size of the contour of the whole surface and of the checkerboard elements at the midpoint of the surface. The texture elements on all surfaces were alternating light (phosphorescent green) and dark squares, like those on a checkerboard. The constant-image-size surfaces prevented observers from simply using the number of square texture elements from the front edge of the surface to judge distance, because targets at the same horizontal distance would entail a different number of texture elements in front of and beyond them and, thus, a different relative position within the total layout of the surface in each case.

\section{Method}

Participants. Thirty-two adults - aged 17-28 years-participated as observers, 8 in each of the four conditions. All had normal or corrected-to-normal vision. All were reimbursed at $\$ 10$ per hour.

Apparatus and Procedure. The apparatus and procedure were the same as those in Experiment 1, except that only horizontal targets were tested, and each target now appeared on a visible checkerboard support surface. In the constant-image-size condition, three different checkerboard patterns were used — one for each eye height — so that the image size of the checkered squares at the center of the surface was constant. This was only tested at the $100-\mathrm{cm}$ eye-height range. Otherwise, a single surface was positioned at each of the three eye heights in the other three conditions - that is, an eye-height range of $50 \mathrm{~cm}$ and $100 \mathrm{~cm}$ in the dark (as shown respectively in Figures 2C and 2D) and an eye-height range of $50 \mathrm{~cm}$ in normal lighting. The checkerboard surfaces were placed on top of the rods used to position targets in Experiment 1 at one of the three eye heights. Each trial tested one of the seven target locations and required the particular support surface to be placed on the support rods and the target to be placed appropriately on the surface while opaque goggles occluded the participant's vision.

The design included three within-subjects factors - horizontal distance (near, medium, and far), HVF (low, medium, high), and repetition (1-5) - and one between-subjects factor, eye-height range $(50 \mathrm{~cm}$ and $100 \mathrm{~cm})$. In addition, the constant-image-size support surfaces were tested with a different group of subjects at the $100-\mathrm{cm}$ eye-height range while normal lighting was also tested with a fourth group of subjects at the 50 -cm eye-height range.

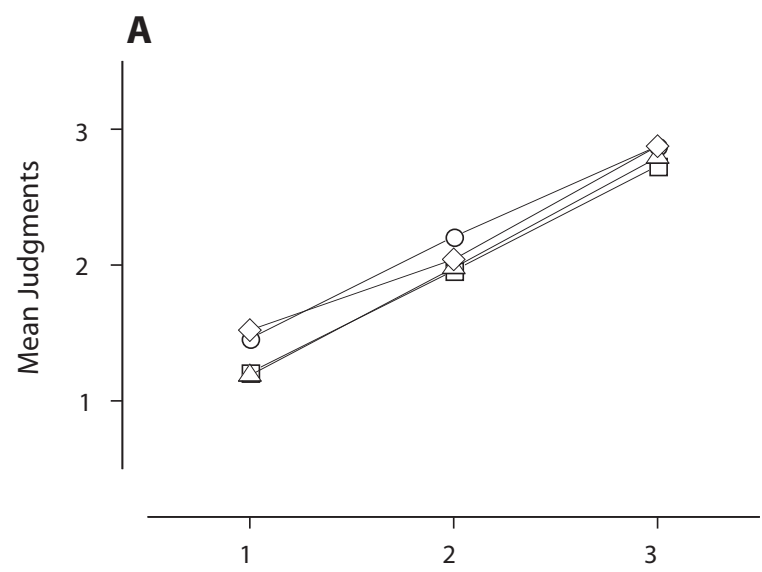

B

Horizontal Distance

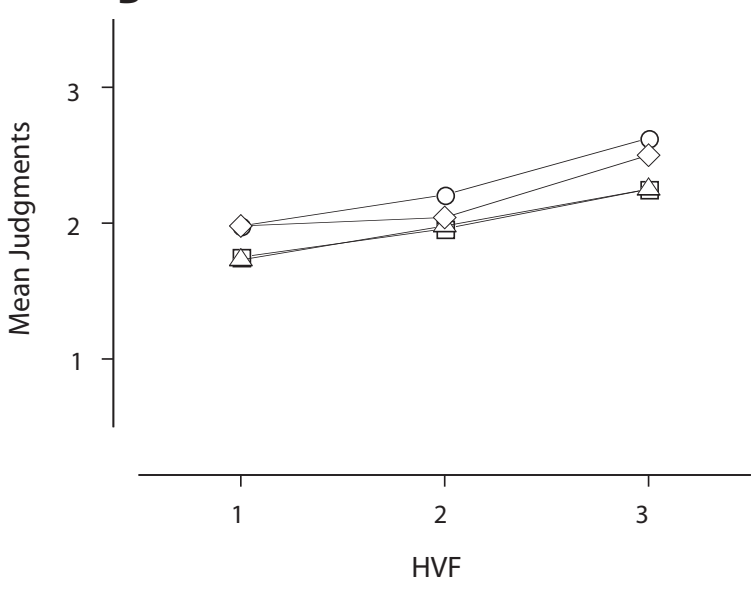

Figure 4. Results of Experiment 2 with visible support surfaces. (A) Judgment means for three targets at a single HVF as a function of horizontal distance. (B) Judgment means for three targets at a single horizontal distance as a function of HVF. Targets in the dark at $50 \mathrm{~cm}$ (open circles) and $100 \mathrm{~cm}$ (open squares), and targets at $50 \mathrm{~cm}$ in the light (open triangles) and in the dark on constant image-size surfaces (open diamonds). 
Table 3

Results of Multiple Regressions Performed on Group Data in Four Conditions of Experiment 2

\begin{tabular}{|c|c|c|c|c|c|}
\hline \multirow[b]{2}{*}{ Condition } & \multirow[b]{2}{*}{$R^{2}$} & \multicolumn{2}{|c|}{$\mathrm{HVF}$} & \multicolumn{2}{|c|}{ Distance } \\
\hline & & Coefficient & Partial $F$ & Coefficient & Partial $F$ \\
\hline $\begin{array}{l}50-\mathrm{cm} \mathrm{EH} \\
\quad \text { (in the dark) }\end{array}$ & $.48^{* * *}$ & $.32^{* * *}$ & 30.9 & $.71^{* * *}$ & 148.7 \\
\hline $\begin{array}{l}100-\mathrm{cm} \text { EH } \\
\text { (in the dark) }\end{array}$ & $.58^{* * * *}$ & $.25^{* * * *}$ & 26.4 & $.76^{* * *}$ & 245.2 \\
\hline $\begin{array}{l}50-\mathrm{cm} \mathrm{EH} \\
\quad \text { (in the light) }\end{array}$ & $.60^{* * *}$ & $.26^{* * *}$ & 28.1 & $.81^{* * *}$ & 269.3 \\
\hline $\begin{array}{l}\text { Constant } \\
\text { image size }\end{array}$ & $.57^{* * * *}$ & $.26^{* * *}$ & 33.7 & $.68^{* * *}$ & 223.0 \\
\hline
\end{tabular}

\section{Results}

As in Experiment 1, we computed means for each of the five target locations that decorrelated HVF and horizontal distance. We also performed multiple regressions on the

Table 4

Results of Multiple Regressions Performed on Individual Observer Data in Four Conditions of Experiment 2

\begin{tabular}{|c|c|c|c|c|c|}
\hline \multirow{2}{*}{$\begin{array}{l}\text { NS } \\
\text { Factor }\end{array}$} & \multirow[b]{2}{*}{$R^{2}$} & \multicolumn{2}{|c|}{ HVF } & \multicolumn{2}{|c|}{ Distance } \\
\hline & & Coefficient & $\overline{\text { Partial } F}$ & Coefficient & Partial $F$ \\
\hline \multicolumn{6}{|c|}{$\begin{array}{l}\text { Visual Support Surface in the Dark } \\
\text { 50-cm Eye Height }\end{array}$} \\
\hline$X$ & $.62^{* * *}$ & .20 & 1.7 & $.90^{* * *}$ & 33.9 \\
\hline & $.67^{* * *}$ & $.70^{* * *}$ & 28.8 & $.30^{*}$ & 6.4 \\
\hline & $.57^{* * * *}$ & $.40^{*}$ & 4.8 & $.90^{* * *}$ & 24.5 \\
\hline & $.66^{* * *}$ & $.40^{* *}$ & 8.5 & $.80^{* * * *}$ & 33.8 \\
\hline $\mathrm{X}$ & $.57^{* * *}$ & .20 & 1.7 & $.80^{* * *}$ & 27.1 \\
\hline$X$ & $.62^{* * *}$ & .10 & 0.7 & $.70^{* * * *}$ & 35.9 \\
\hline$X$ & $.65^{\text {*** }}$ & .20 & 1.9 & $.90^{* * * *}$ & 39.2 \\
\hline & $.50^{* * *}$ & $.40^{* *}$ & 8.5 & $.50^{* *}$ & 13.3 \\
\hline \multicolumn{6}{|c|}{ 100-cm Eye Height } \\
\hline & $.31^{*}$ & $.50^{*}$ & 5.9 & $.40^{*}$ & 7.4 \\
\hline & $.67^{* * *}$ & $.10^{* *}$ & 13.8 & $.60^{* * *}$ & 30.9 \\
\hline$X$ & $.92^{* * * *}$ & .10 & 2.6 & $1.0^{\text {*** }}$ & 255.8 \\
\hline & $.67^{* * *}$ & $.30^{*}$ & 5.4 & $.80^{* * *}$ & 38.5 \\
\hline & $.55^{* * *}$ & $.40^{*}$ & 6.6 & $.70^{* * *}$ & 20.2 \\
\hline$X$ & $1.0^{* * *}$ & .00 & 0 & $1.0^{* * *}$ & 229.1 \\
\hline$X$ & $.46^{* * *}$ & .30 & 3.7 & $.60^{* * *}$ & 14.8 \\
\hline $\mathrm{X}$ & $1.0^{* * *}$ & .00 & 0 & $1.0^{* * *}$ & 229.1 \\
\hline \multicolumn{6}{|c|}{ Support Surface in the Light } \\
\hline \multirow{5}{*}{$\mathrm{X}$} & $.92^{* * * *}$ & $.30^{*}$ & 9.6 & $1.0^{* * *}$ & 106.8 \\
\hline & $.44^{* *}$ & $.40^{*}$ & 8.5 & $.40^{* *}$ & 8.5 \\
\hline & $.36^{* *}$ & .10 & 0.3 & $.60^{* *}$ & 12.2 \\
\hline & $.90^{* * *}$ & $.70^{* * *}$ & 62.0 & $1.0^{* * *}$ & 126.4 \\
\hline & $.76^{* * *}$ & $.50^{* * *}$ & 28.9 & $.60^{* * *}$ & 41.7 \\
\hline$X$ & $.83^{* * * *}$ & -.10 & 1.3 & $.90^{* * *}$ & 108.7 \\
\hline$X$ & $.92^{* * *}$ & .10 & 2.5 & $1.0^{* * *}$ & 255.8 \\
\hline $\mathrm{X}$ & $.92^{* * *}$ & .10 & 2.5 & $1.0^{* * *}$ & 255.8 \\
\hline \multicolumn{6}{|c|}{$\begin{array}{l}\text { Visible Constant-Image-Size } \\
\text { Surface in the Dark }\end{array}$} \\
\hline & $.79^{* * *}$ & $.50^{* * *}$ & 33.1 & $.60^{* * *}$ & 47.7 \\
\hline & $.64^{* * *}$ & $.30^{*}$ & 7.8 & $.60^{* * * *}$ & 31.1 \\
\hline$X$ & $.66^{* * * *}$ & .20 & 3.2 & $.70^{* * * *}$ & 39.9 \\
\hline$X$ & $.82^{* * * *}$ & .00 & 0 & $.80^{* * * *}$ & 97.8 \\
\hline \multirow[t]{3}{*}{$X$} & $.50^{* * *}$ & .20 & 1.6 & $.70^{* * * *}$ & 20.2 \\
\hline & $.66^{* * *}$ & $.40^{* * *}$ & 11.4 & $.50^{* * *}$ & 25.7 \\
\hline & $.68^{* * *}$ & $.30^{*}$ & 7.2 & $.70^{* * *}$ & 39.1 \\
\hline$X$ & $.69^{* * *}$ & .20 & 2.9 & $.80^{* * * *}$ & 46.3 \\
\hline
\end{tabular}

Note-HVF, height in the visual field. ${ }^{*} p<.05 .{ }^{* *} p<.01 .{ }^{* * *} p<$ .001 . combined data of the observers in each condition, regressing HVF and horizontal distance on verbal distance judgments. As shown in Figure 4 and Table 3, the results in all four conditions were essentially the same. Judgments covaried strongly with horizontal distance (coefficients $\approx$ .70 in the dark and $\approx .80$ in normal lighting). There was a tendency to overestimate near-target distance and underestimate far-target distance. The group responses varied weakly with HVF (coefficients $\approx .30$ ).

Next, we performed multiple regressions on the data for each observer in each condition. In all four conditionsas shown in Table 4- HVF failed to reach statistical significance for half of the observers, whereas distance was significant for all observers, with most coefficients being between .60 and 1.00. Essentially, the pattern of results in Experiment 1 was reversed in Experiment 2.

In summary, the group data show that distance was now judged more accurately on average. The size-distance paradox effect disappeared, and the influence of HVF was much diminished. Moreover, examination of the individual data revealed that half of the participants were not influenced by HVF, especially in normally lighted conditions. Thus, when optical structure from a visible support surface was introduced, observers' judgments started to show no influence of HVF. Finally, the results were replicated in the dark, in normal lighting, and in the dark with constantimage-size surfaces. This latter result showed that observers were not merely using the number of visible texture elements between the front edge of the surfaces and the targets to judge distance, nor were they using the relative position within the visible contours of the surfaces.

\section{EXPERIMENT 3}

In the first two experiments, we used a configuration of seven targets allowed by the intersection of three horizontal distances, three eye heights, and three vertical-gaze angles (or HVF). This configuration left the far location on the highest support surface as well as the nearest location on the lowest surface unused. It is possible that participants expected some far and near targets there, respectively. If so, this would explain the tendency for HVF to

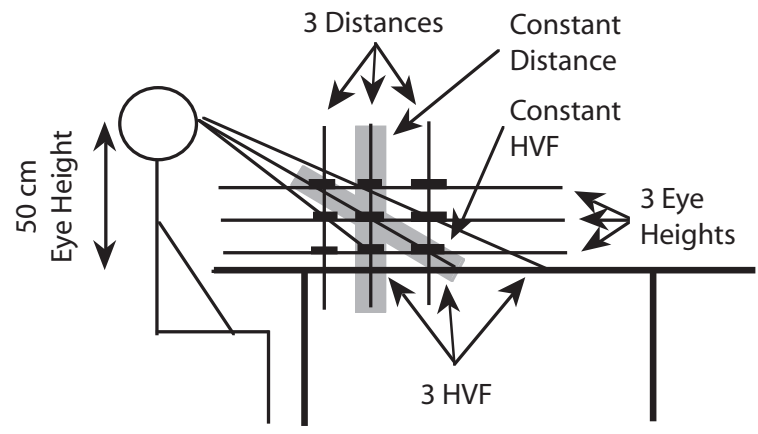

Figure 5. Arrangement of the targets in Experiment 3 that was similar to those of the previous experiments, except two more targets were added at the lower left and upper right. The extra targets did not fall along one of the three HVF. 
influence distance judgments in some participants once there was a visible support surface at each eye height. We retested the $50-\mathrm{cm}$ eye-height range with a visible support surface in the dark and in normal lighting, and included the full nine target locations - as shown in Figure 5-in order to address this issue.

\section{Method}

Participants. Eight adults - aged 18-25 years-participated as observers. All had normal or corrected-to-normal vision. All were reimbursed at $\$ 10$ per hour.

Apparatus and Procedure. These were identical to the dark and normally lighted viewing conditions of Experiment 2, except that nine target locations were tested, including a far target at the smallest eye height (highest support surface) and a near target at the largest eye height (lowest support surface). The former added an additional HVF that would specify a farthest target, and the latter added yet another HVF that would specify a nearest target. Both
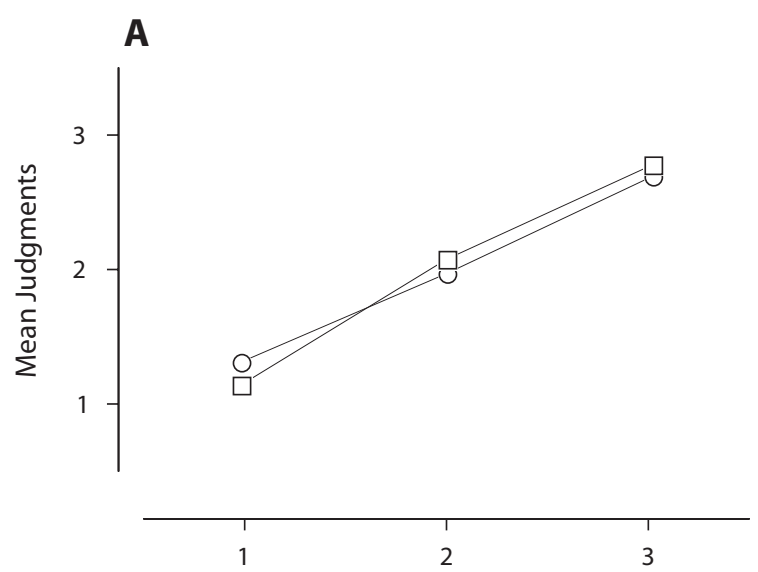

B

Horizontal Distance

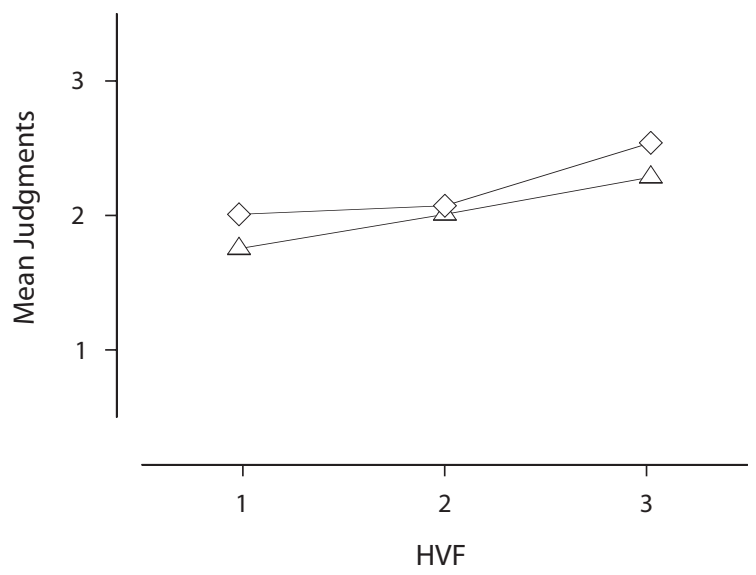

Figure 6. Results of Experiment 3 with visible support surfaces and nine target locations. (A) Judgment means for three targets at a single HVF as a function of horizontal distance. (B) Judgment means for three targets at a single horizontal distance as a function of HVF. Targets at $50 \mathrm{~cm}$ in the dark (open circles in [A] and open triangles $[\mathrm{B}]$ ) and in the light (open squares in $[\mathrm{A}]$ and open diamonds in $[\mathrm{B}])$.
Table 5

Results of Multiple Regressions Performed on

Group Data in Two Conditions of Experiment 3

\begin{tabular}{|c|c|c|c|c|c|}
\hline \multirow[b]{2}{*}{ Condition } & \multirow[b]{2}{*}{$R^{2}$} & \multicolumn{2}{|c|}{ HVF } & \multicolumn{2}{|c|}{ Distance } \\
\hline & & Coefficient & $\overline{\text { Partial } F}$ & Coefficient & Partial $F$ \\
\hline Dark & $.43^{* * *}$ & $.11^{*}$ & 3.9 & $.68^{* * * *}$ & 143.5 \\
\hline Light & $.68^{* * *}$ & $.15^{\text {*** }}$ & 14.1 & $.80^{* * *}$ & 401.0 \\
\hline
\end{tabular}

Table 6

Results of Multiple Regressions Performed on Individual Observer Data in Two Conditions of Experiment 3

\begin{tabular}{|c|c|c|c|c|c|}
\hline \multirow{2}{*}{$\begin{array}{l}\mathrm{NS} \\
\text { Factor }\end{array}$} & \multirow[b]{2}{*}{$R^{2}$} & \multicolumn{2}{|c|}{ HVF } & \multicolumn{2}{|c|}{ Distance } \\
\hline & & Coefficient & Partial $F$ & Coefficient & Partial $F$ \\
\hline \multicolumn{6}{|c|}{ Dark } \\
\hline$X$ & $1.0^{* * *}$ & .00 & 0.0 & $1.0^{* * *}$ & big \\
\hline$X$ & $.42^{* *}$ & .00 & 0.0 & $.50^{* * *}$ & 15.7 \\
\hline$X$ & $.80^{* * * *}$ & .00 & 0.0 & $.80^{* * *}$ & 88.0 \\
\hline $\mathrm{X}$ & $.53^{* * *}$ & -.10 & 0.3 & $.90^{* * *}$ & 24.9 \\
\hline$X$ & $.41^{* *}$ & .00 & 0.0 & $.50^{* * *}$ & 15.0 \\
\hline \multirow[t]{2}{*}{$X$} & $.35^{* *}$ & .00 & 0.0 & $.70^{* *}$ & 12.1 \\
\hline & $.57^{* * * *}$ & $.40^{*}$ & 7.2 & $.70^{* * *}$ & 22.2 \\
\hline$X$ & $.46^{* *}$ & $.60^{* * *}$ & 14.8 & .30 & 3.7 \\
\hline \multicolumn{6}{|c|}{ Light } \\
\hline X & $.77^{* * *}$ & .10 & 0.9 & $.90 * * *$ & 73.0 \\
\hline$X$ & $.34^{* *}$ & .10 & 0.7 & $.40^{* *}$ & 10.8 \\
\hline \multirow[t]{3}{*}{$\mathrm{X}$} & $1.0^{* * *}$ & .00 & 0.0 & $1.0^{* * * *}$ & big \\
\hline & $.88^{* * *}$ & $.20^{*}$ & 6.1 & $1.0^{* * *}$ & 152.8 \\
\hline & $.73^{* * *}$ & $.40^{* * *}$ & 22.0 & $.50^{* * *}$ & 34.4 \\
\hline$X$ & $1.0^{* * *}$ & .00 & 0.0 & $1.0^{* * *}$ & big \\
\hline \multirow[t]{2}{*}{$X$} & $.83^{* * *}$ & .00 & 0.0 & $1.0^{* * *}$ & 110.0 \\
\hline & $.70^{* * *}$ & $.60^{* * *}$ & 15.7 & $.40^{* * *}$ & 35.4 \\
\hline
\end{tabular}
001 .

dark and lighted conditions were tested in succession and in that order within subjects.

\section{Results}

We computed means for each target location, and, as shown in Figure 6, mean judgments varied primarily with horizontal distance. The mean judgments for the three HVF (including the additional one, marked by an asterisk) at the near-target location (combining dark and light conditions) were $1.08^{*}, 1.08$, and 1.18. Similarly, at the far target location, the means were $2.66,2.81$, and $2.85^{*}$. Thus, the two additional HVF did not yield judgments that were more strongly near or far, respectively. Multiple regressions on the group data yielded coefficients for distance of .70 in the dark and of .80 in normal lighting, replicating the results in Experiment 2. The coefficients for HVF were smaller $(\approx .13)$, although still statistically significant, as shown in Table 5. Multiple regressions performed separately on the data for each observer in each condition showed that most observers were not influenced by HVF. As shown in Table 6, 6 of the 8 observers in the dark condition and 5 in the lighted condition yielded coefficients and partial $F \mathrm{~s}$ for HVF that were either equal to or near 0 . One observer's judgments were influenced primarily by HVF, and two others showed a slight effect. Otherwise, 
observers were not affected by the variations in HVF and thus judged horizontal distance correctly.

Once more, the group data suggested that HVF was influencing the participants' responses. However, examination of the individual data showed that in fact, most observers were indifferent to changes in HVF.

\section{GENERAL DISCUSSION}

In Experiment 1, we explored verbal distance reports in sparse viewing environments when HVF was isolated as the only really salient cue available for gauging target distance. The apparatus was arranged in such a way that HVF altered across target position, but the HVF changes did not convey useful information because the targets were positioned at different eye heights. In the introduction, we predicted that the participants would reliably misjudge the distance of three targets lying in the same frontoparallel plane but at different heights. We expected that judgments would covary with HVF because we arranged the displays so that HVF would be the only salient cue available. However, it was not possible to eliminate all alternative cues. Because accommodation also remained, we predicted a counterintuitive pattern of results on the basis of the size-distance-paradox phenomenon, as described previously (Mon-Williams \& Tresilian, 1999). That is, we predicted that the arrangement (see Figure 1) would produce a (weak) negative correlation with the horizontal target distance. The results of Experiment 1 were in line with these a priori predictions. These results were replicated at 50 - and $100-\mathrm{cm}$ viewing heights and with target surfaces of both frontoparallel and horizontal orientations.

Thus, we found that observer performance in an isolated cue task demonstrated that observers could detect and use a hypothesized cue - HVF - to judge a perceptible property, horizontal distance. Is it safe for one to infer that the cue is used in representative multicue situations? Perhaps not. Analysis and description of the cue might simply be incorrect; that is, the cue might be related in some way to information actually used without being identical to that information. Thus, when the cue is isolated, observers might detect and use it in lieu of anything else. However, when observers are provided with normally available information, they might well use that information and not the cue. However, because the cue may covary with the information actually used, it may appear that the cue is being used in multicue contexts.

The primary question of interest within our study was how participants' verbal responses would alter when the participants could see a visible supporting surface. In Experiment 2, more surface-relative information was made available. The targets were positioned at different distances along the support surfaces that were now visible at each of the three different eye heights. In these conditions, observers judged horizontal distance more accurately. In the group data, observers seemed to continue to be influenced by HVF. Nonetheless, analysis of individual participant data revealed that half of the partic- ipants were not affected by HVF at all. In Experiment 3, we extended the number of testing locations in order to see whether this manipulation would further decrease the influence of HVF on participants' responses. We retested the 50-cm eye-height range with a visible support surface in both the dark and normal lighting and included nine target locations. The group data from Experiment 3 were similar to those of Experiment 2. However, analyses of the individual data showed that only two of the participants' responses were biased by HVF in this experiment. Thus, the results of Experiments 2 and 3 showed clearly that the simple addition of a visible support surface was sufficient to eliminate the influence of HVF on judgments of nearly all observers and to greatly increase the accuracy of their verbal responses.

We set up our experimental displays to be representative of the environmental structure confronted by observers when evaluating the distances of objects supported on surrounding surfaces. These support surfaces are typically at a variety of different eye heights. In common indoor environments-like an office, someone's living room, or a classroom-objects will be seen on support surfaces (desks, coffee and end tables, chairs and benches, fireplace mantels, flower stands, shelves, the floor, etc.) arrayed at many different eye heights. Similarly, in outdoor environments, the surfaces of walls, raised walkways, tree limbs, stairways, nonlevel surfaces of the ground, and the like also support objects at variable eye heights. These circumstances make it clear that information projected from the relevant support surfaces is essential for reliable information about object distances.

The informative value of the classic HVF cue is derived from the assumed structure of a support surface. Under conditions of objects on a simple flat-ground surface, HVF will covary perfectly with distance and, as such, could form part of a complex information variable that includes optical structure from surface. In fact, given any one of the surfaces in our experiment at a given eye height, the HVF may well have been a coherent part of the information that observers used to judge distances. In this case, HVF is merely incomplete as a specification of the information used to perceive distance. With a visible support-surface structure, the better alternative is to find and use information about the support surface projected from its actual structure. HVF - as a derived variable that leaves the surface structure implicit-is bound to be less reliable. To the extent that HVF leaves the supporting surface structure entirely implicit, it is further derived and even more remote from the motivating environmental structure (Gardner \& Mon-Williams, 2001; Wallach \& O’Leary, 1982).

There is an ontological problem here with respect to information. When might two things actually be one thing? A pattern described as two different cues might in fact be parts of a single coherent pattern and thus compose a single cue, since presumably the aspects that might be differentiated typically covary, given that they specify the same thing. The argument for the use of multiple cues 
is, in part, that the composition of multiple cues provides better information because it covaries more precisely with the corresponding perceptible property. The point is that what is obtained is a different variable that actually specifies something different than what is specified by either cue alone. The unique aspect of the design of these experiments is that we were able to show that the simple variable-HVF or vertical gaze angle-was no longer really viable in the more complex and complete information context, and as such, it was not used. Nevertheless, it remains possible that HVF or vertical-gaze angle was a part of another, more complex variable that enabled observers to correctly evaluate the distances of target objects on surfaces at different eye heights. HVF may merely be an analytically distinguishable part of optical pattern that specifies object distance in the context of (and in terms of) support surface layout (i.e., support surface shape, orientation, and eye height). The mistake is to overanalyze and, as a result, hypothesize that HVF is available and used as a separate variable. Probabilistic approaches to information set out to provide an estimate of the degree of covariation between given hypothetical cues and corresponding world properties. Imperfect correspondence is then used to predict imperfect responding; that is, if the cue predicts the world property with modest probability, then the cue is predicted to be weighted modestly (see, e.g., Brunswik, 1952; Knill \& Richards, 1996). The problem is that the imperfection may reside not in the variables normally used by the visual system, but in the scientist's individuation of potential information variables.

The methodological problem that accompanies this theoretical problem is that the visual system is highly adaptive. Braunstein and Todd (1990) have noted that observers will use whatever optical structure they can find in experimental tasks to enable them to perform within specified constraints. When only a single optical property varies with the assigned task variable, observers might well use that variable in a reliable way, but when better information is made available, they use it instead. Such adaptability and flexibility might also be expected to produce significant individual differences in circumstances involving more complex information. Indeed, we observed such individual differences. Ultimately, individual data and results had to be examined in order to properly understand what observers were doing. Given the normal covariation of HVF and surfacerelative information, one might well have inferred on the basis of the present group results that HVF was reliably used in representative conditions. The unique design in these experiments showed that this inference would be incorrect.

The results were replicated at both $50-\mathrm{cm}$ and $100-\mathrm{cm}$ viewing heights, and with both dark and lighted viewing conditions and, finally, with a constant-image-size support surface. Of course, image sizes could not be held constant along the full extent of these constant-image-size surfaces without distorting essential information about surface structure (and this is partly the point here). Only the image sizes at a locus along the surfaces - in this case, the midpoint - could be held constant in addition to the image size of the entire surface contour. This stopped observers from judging distance in terms of the number of surface texture elements (checkerboard squares) from the front edge of the surface or in terms of the relative position of the target within the contour of the visible surface. Nevertheless, these visible support surfaces were found to be effective in enabling observers to judge target distances, despite differences in eye height. The information about distance projected from these visible support surfaces was necessarily more abstract than the countable texture elements along the support surfaces. (See Bingham \& Shull [2000, 2001] for a description of the distance information available from such surface structure and for supportive evidence.)

In summary, the results of the present study emphasize the importance of considering the role of supporting surfaces in distance perception. The findings highlight fundamental problems when considering the classic distance cue HVF independent of the relevant support surfaces. More fundamentally, the study raises the conundrum that if cues are assumed to relate to corresponding perceptible properties of the world in a probablistic or partial manner, then researchers are left at a serious disadvantage. Investigators are left without criteria for deciding when they have successfully described distinct information variables used by the visual system.

\section{AUTHOR NOTE}

Address correspondence to G. P. Bingham, Department of Psychology, Indiana University, 1101 E. 10th St., Bloomington, IN 47405-7007 (e-mail: gbingham@indiana.edu).

\section{REFERENCES}

Bingham, G. P., \& Shull, J. A. (2000, November). Distance information in eyeheight units from texture gradients: Theory and data. Paper presented at the annual meeting of the Psychonomic Society, New Orleans.

Bingham, G. P., \& Shull, J. A. (2001, July). Role of visible support surfaces in calibrating visual information used to guide reaches. Paper presented at the 11th International Conference on Perception and Action, Storrs, CT.

Braunstein, M. L., \& TodD, J. T. (1990). On the distinction between artifacts and information. Journal of Experimental Psychology: Human Perception \& Performance, 16, 211-216.

Bruno, N., \& Cutting, J. E. (1988). Minimodularity and the perception of layout. Journal of Experimental Psychology: General, 117, 161-170.

BRUNSwIK, E. (1952). The conceptual framework of psychology. Chicago: University of Chicago Press.

Collett, T. S., \& Udin, S. B. (1988). Frogs use retinal elevation as a cue to distance. Journal of Comparative Physiology A, 163, 677-683.

Cutting, J. E. (2003). Reconceiving perceptual space. In H. Hecht, M. Atherton, \& R. Schwartz (Eds.), Looking into pictures: An interdisciplinary approach to pictorial space (pp. 215-238). Cambridge, MA: MIT Press.

Cutting, J. E., \& Vishton, P. M. (1995). Perceiving layout and knowing distances: The integration, relative potency, and contextual use of different information about depth. In W. Epstein \& S. Rogers (Eds.), Perception of space and motion (pp. 69-117). San Diego: Academic Press.

EMmerT, E. (1881). Grössenverhältnisse der Nachbilder: Klinische Monatsblätter für Augenheilkunde, 19, 443-450.

Epstein, W. (1966). Perceived depth as a function of relative height 
under three background conditions. Journal of Experimental Psychology, 72, 335-338.

Fisher, S. K., \& CiUfFredA, K. J. (1988). Accommodation and apparent distance. Perception, 17, 609-621.

Gardner, P., \& Mon-Williams, M. (2001). Vertical gaze angle: Absolute height-in-scene information for the programming of prehension. Experimental Brain Research, 136, 379-385.

Gillam, B. (1995). The perception of spatial layout from static optical information. In W. Epstein \& S. Rogers (Eds.), Perception of space and motion (pp. 23-67). San Diego: Academic Press.

Knill, D. C., \& Richards, W. (1996). Perception as Bayesian inference. Cambridge: Cambridge University Press.

Landy, M. S., Maloney, L. T., Johnston, E., \& Young. M. J. (1995). Measurement and modeling of depth cue combination: In defense of weak fusion. Vision Research, 35, 389-412.

Mon-Williams, M., McIntosh, R. D., \& Milner, A. D. (2001). Vertical gaze angle as a distance cue for programming reaching: Insights from visual form agnosia II (of III). Experimental Brain Research, 139, 137-142.

Mon-Williams, M., \& Tresilian, J. R. (1999). The size-distance para- dox is a cognitive phenomenon. Experimental Brain Research, 126, 578-582.

Mon-Williams, M., \& Tresilian, J. R. (2000). An ordinal role for accommodation in distance perception. Ergonomics, 43, 391-404.

OoI, T. L., Wu, B., \& He, Z. J. (2001). Distance determined by angular declination below the horizon. Letters to Nature, 414, 197-200.

Philbeck, J. W., \& Loomis, J. M. (1997). Comparison of two indicators of perceived egocentric distance under full-cue and reduced-cue conditions. Journal of Experimental Psychology: Human Perception \& Performance, 23, 72-85.

ScHIFF, W. (1980). Perception: An applied approach. Boston: Houghton Mifflin.

SedGwick, H. A. (1986). Space perception. In K. R. Boff, L. Kaufman, \& J. P. Thomas (Eds.), Handbook of perception and human performance (pp. 1-57). New York: Wiley.

Wallach, H., \& O'Leary, A. (1982). Slope of regard as a distance cue. Perception \& Psychophysics, 31, 145-148.

(Manuscript received September 7, 2006; revision accepted for publication November 7, 2007.) 